\title{
INPOP06: a new numerical planetary ephemeris
}

\author{
A. Fienga ${ }^{1,2}$, H. Manche ${ }^{1}$, J. Laskar $^{1}$, and M. Gastineau ${ }^{1}$ \\ 1 Astronomie et Systèmes Dynamiques, IMCCE-CNRS UMR8028, 77 Av. Denfert-Rochereau, 75014 Paris, France \\ 2 Observatoire de Besançon-CNRS UMR6213, 41 bis Av. de l'Observatoire, 25000 Besançon, France \\ e-mail: agnes@obs-besancon.fr
}

Received 20 October 2006 / Accepted 17 September 2007

\section{ABSTRACT}

\begin{abstract}
INPOP06 is the new numerical planetary ephemeris developed at the IMCCE-Observatoire de Paris. INPOP (Intégrateur Numérique Planétaire de l'Observatoire de Paris) is a numerical integration of the motion of the nine planets and the Moon fitted to the most accurate available planetary observations. It also integrates the motion of 300 perturbing main belt asteroids, the rotation of the Earth and the Moon libration. We used more than 55000 observations including the latest tracking data of the Mars Global Surveyor (MGS) and Mars Odyssey (Odyssey) missions. The accuracy obtained with INPOP06 is comparable to the accuracy of recent versions of the JPL DE ephemerides (DE414, Standish 2003, JPL IOM, 312N, 03; Konopliv et al. 2006, Icarus, 182, 23) and of the EPM ephemerides (EPM2004, Pitjeva 2005, Sol. Syst. Res., 39, 176).
\end{abstract}

Key words. celestial mechanics - ephemerides - astrometry

\section{Introduction}

The launch by NASA of the first interplanetary missions is a part of a considerable and continuous effort to develop and improve planetary ephemerides. The Jet Propulsion Laboratory (JPL) was entrusted with this task and produced many ephemerides combining the best theories and the most recent observational techniques, such as range measurements or VLBI tracking. Major improvements in observational accuracy (Lunar Laser Ranging, range and VLBI spacecraft tracking) permitted by modern technology, and in response to more demanding needs, have led to comparable improvements in the accuracy of the planetary and lunar ephemerides. Based on some first versions of the numerical integration of planetary motions (see for instance Devine \& Dunham 1966; Ash et al. 1971), the DE96 JPL ephemerides (Standish et al. 1976) were among the first of the known and widely distributed accurate numerical ephemerides fitted to observations developed by JPL. These were followed by DE200 (Standish 1990), DE403 (Standish et al. 1995) and DE405 (Standish 1998). All these ephemerides are numerically integrated with a variable step-size, variable-order, Adams method. Their dynamical model includes point-mass interactions between the nine planets, the Sun and asteroids, relativistic PPN effects (Moyer 1971, 2000), figure effects, Earth tides and lunar librations (Newhall et al. 1983). Since DE96, some improvements have been added to the DE ephemerides, and new ephemerides such as DE409 (Standish 2004), DE410 (Standish 2005) and DE414 (Standish 2003; Konopliv et al. 2006) were constructed and fitted on increasingly dense sets of space mission tracking data. Numerical solutions have also being developed at the Institute of Applied Astronomy of the Russian Academy of Sciences (IAA RAS). They are based on a dynamical model very similar to the JPL one. These ephemerides, EPM, are also fitted to optical, radar and space tracking data and have an accuracy comparable to the JPL ephemerides (Krasinsky et al. 1982; 1986; 1993; Pitjeva 2001, 2005).
For many years, the accurate planetary ephemerides built at the JPL have been the only source of numerical ephemerides readily available. Besides the two numerical ephemerides presented above, the IMCCE has developed, since the early 80s, some analytical ephemerides of the planetary motion. However, these ephemerides do not have enough accuracy for comparison with the latest and more accurate spacecraft tracking observations. Especially for Mars, the intrinsic accuracy of the analytical ephemerides (series limitation accuracy) is about $100 \mathrm{~m}$ over 30 years (Fienga \& Simon 2005). Over the same interval of time the mean accuracy of Viking, Pathfinder and MGS tracking data is of a few meters. It appears thus that these analytical ephemerides are not accurate enough to be used in modern data analysis of space missions.

Consequently, a new aspect of IMCCE planetary ephemerides evolution arose with the development of a numerical ephemeris of planet motion called INPOP (Intégration Numérique Planétaire de l'Observatoire de Paris). This project started in 2003 from the needs of short term accuracy ephemerides for the analysis of Earth-based and space mission observational data but also from the necessity for improvements in the dynamical model for the long term astronomical ephemerides used in paleoclimate studies of the Earth and Mars over several millions of years. Indeed, because of the chaotic behavior of the orbital solutions of the Solar System (Laskar 1989, 1990), extending the astronomical solutions from $40 \mathrm{Myr}$ (Laskar et al. 2004a,b) to $60 \mathrm{Myr}$ corresponds to a gain of two orders of magnitude in the precision of the model and parameters. For these reasons, IMCCE decided to develop a new numerical planetary ephemeris adjusted to space mission tracking observations. INPOP has to be accurate over very short periods of time, but must also be extended over very long time intervals of several million years.

In this paper, we present the latest version of our short-term ephemeris INPOP, INPOP06. We describe the dynamical model used for the integration of the planets and Moon motion and 
the Earth and Moon rotation. We compare our model with the DE405 ephemerides (Standish 1998). This first step is important for the validation of our study, as we demonstrate that we can recover the DE405 ephemeris in a very precise way. In the second part, we describe the fit made to observations. We present the observation processing and the residuals obtained with INPOP. The observation residuals depend on the models used in the reduction procedures and on the parameters that are determined in the fit. For the present determination, we have chosen to minimize the number of fitted parameters. This explains why different and better residuals are presented in Konopliv et al. (2006) and Pitjeva (2005).

Two INPOP versions are obtained: the first one, INPOP05, mimicking DE405 in the dynamical model and data fit, and a second, INPOP06, developed independently and fitted on data available untill early 2006. New determinations of physical parameters such as asteroid masses and Sun oblateness are presented and compared to other values found in the literature.

\section{The INPOP dynamical model}

\subsection{General features}

INPOP is a numerical integration of the equations of motion of the planets of our Solar System. It is also a numerical integration of the Earth orientation and Moon rotation. Besides the classic planet equations of motion given in Moyer (1971), specific developments were performed especially related to the Mars motion, Earth rotation and the Moon libration. A special effort was paid to the minimization of the roundoff errors during the integration processes. The integrator is an Adams-Cowell method with fixed step-size, and the programming is done in $\mathrm{C}$ language, thus allowing to use the extended precision ( 80 bits) on Intel Itanium II processors.

The development strategy was to build in a first stage a solution (called INPOP05) as close as possible to the DE405 JPL ephemeris. INPOP05 was constructed as a test bed to demonstrate our capabilities in planetary ephemerides computing and to understand as much as possible the rotation and orbital motion equations used in DE405. Some small differences still remain between the two ephemerides. Indeed, different choices were made in asteroid perturbation computations, in deformation of the Earth due to tide effects, and in the computations of the positions and velocities of the Sun versus the Solar System barycenter. Estimations of the differences are presented in Sect. 2.3.6. An INPOP05 ephemeris fitted to the same set of observations as DE405 will be presented in Sect. 4.2.

In a second stage, a new dynamical model (INPOP06) was developed, following our best understanding of the dynamical equations. Section 2.4 introduces the INPOP06 dynamical model and Sect. 5 presents the INPOP06 fit to observations.

\subsection{INPOP numerical integrator}

The numerical integrations in INPOP are performed with a classical Adams PECE method of order 12 (e.g. Hairer et al. 1993) with the aim to reduce the roundoff error. For this, we have switched to extended precision on Intel architecture. The floating operations then use the 80 bits (with 64 bits mantissa) of the arithmetical unit instead of 64 bits (with 53 bits mantissa) for double precision. The improvement is very significant (Fig. 1) while the CPU cost is nearly the same (Markstein 2000). In Fig. 1, the errors in the computation of the Moon longitude after 100 years are computed for various step sizes by comparison

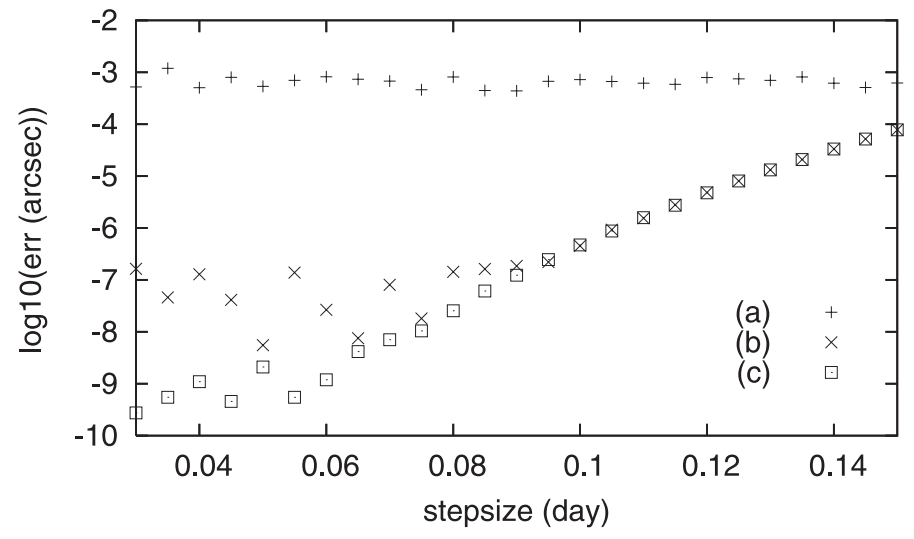

Fig. 1. Numerical error in the Moon longitude (in arcsecond) after 100 years for various settings: a) double precision, b) extended precision, c) extended precision with a corrector step in simulated quadruple precision.

with a very accurate solution obtained with the ODEX numerical integrator in quadruple precision and internal error set to $1 \mathrm{E}-28$ (Hairer et al. 1993).

Integrating in quadruple precision would of course reduce the round off error to a very large amount, but the CPU time is about 15 time greater than for double precision arithmetic (or extended arithmetic) on our machine (Itanium II with Intel C++ compiler). Nevertheless, it was possible to obtain an additional order of magnitude improvement by using a single addition in simulated quadruple precision in the corrector step with a very small overhead (Fig. 1).

For the final integrations, the step size is chosen in order to minimize the roundoff error. For this, we take the largest step size for which the error is dominated by the roundoff error and not by truncation error. This is monitored on the Moon longitude, for which the numerical error is the largest (Fig. 1). The final step size for INPOP06 has been chosen to 0.055 days, but during the fitting procedure, in order to improve CPU time, a 0.1 day step size was preferred.

Finally, with the chosen step size of 0.055 days, the numerical error for all planets has been estimated by an integration made over 10000 years one way and back. The results are displayed in Table 1. In this table, the error over 100 years is estimated by comparison to a high precision integration in quadruple precision with the ODEX integrator, while over 10000 years, the estimate of the error is the half of the difference obtained after integrating one way and back. Even over 10000 years, for most of the planets, the numerical error is so small that it has not reached an asymptotic behavior. For the Moon, the error in longitude behaves as $t^{1.46}$ (Fig. 2), following the optimal Brouwer's law in $t^{3 / 2}$ (Brouwer 1937; Quinn \& Tremaine, 1990).

The analysis of the integration error over time intervals longer than 10000 years is beyond the scope of this paper that is devoted to high accurate planetary ephemerides for astronomical observations and space mission design. One can thus consider that over the time span considered here (10000 years), with our numerical integrator design, the numerical error is negligeable (Table 1).

\subsection{INPOP05 dynamical model: differences with DE405}

Based on the equations developed by Moyer (1971) and used in the construction of the JPL DE numerical ephemerides, we 
Table 1. Estimated numerical error in longitude in INPOP06 (step size 0.055 day) for all planets $i$ ( $i=3$ stands for the Earth-Moon Barycenter, and $i=10$ for the Moon). The error $\varepsilon_{i}$ is given in microarcseconds ( $\mu$ as) or micrometers $(\mu \mathrm{m})$ over 100 years and over 10000 years.

\begin{tabular}{r|lr|lr}
\hline \hline & \multicolumn{2}{|c}{$100 \mathrm{yr}$} & \multicolumn{2}{c}{$10000 \mathrm{yr}$} \\
$i$ & $\varepsilon_{i}(\mu \mathrm{as})$ & $\varepsilon_{i}(\mu \mathrm{m})$ & $\varepsilon_{i}(\mu \mathrm{as})$ & $\varepsilon_{i}(\mu \mathrm{m})$ \\
\hline 1 & $3.3 \times 10^{-4}$ & 93.3 & $1.5 \times 10^{-1}$ & 41288 \\
2 & $1.4 \times 10^{-5}$ & 7.5 & $9.3 \times 10^{-3}$ & 4901 \\
3 & $1.9 \times 10^{-5}$ & 14.0 & $7.3 \times 10^{-3}$ & 5335 \\
4 & $3.0 \times 10^{-6}$ & 3.4 & $4.2 \times 10^{-4}$ & 461 \\
5 & $1.5 \times 10^{-7}$ & 0.6 & $9.0 \times 10^{-6}$ & 34 \\
6 & $2.7 \times 10^{-8}$ & 0.2 & $5.3 \times 10^{-6}$ & 37 \\
7 & $3.9 \times 10^{-7}$ & 5.5 & $1.7 \times 10^{-6}$ & 23 \\
8 & $1.4 \times 10^{-7}$ & 3.1 & $1.3 \times 10^{-6}$ & 29 \\
9 & $7.7 \times 10^{-8}$ & 2.2 & $1.3 \times 10^{-6}$ & 38 \\
10 & $5.1 \times 10^{-4}$ & 1.0 & 1.3 & 2513 \\
\hline
\end{tabular}

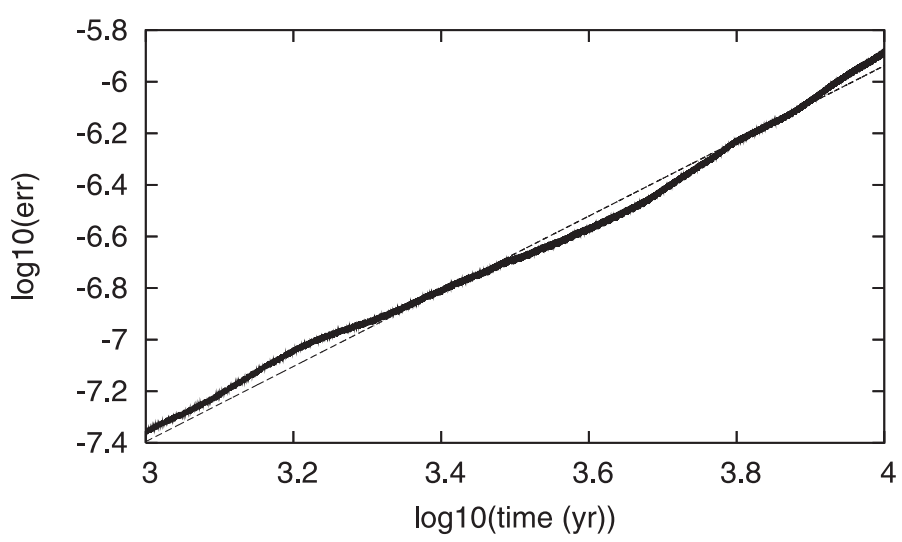

Fig. 2. Evolution of the roundoff error in the longitude of the Moon. The error (in arcsec) is estimated as half of the difference after one way and back over 10000 years. The dashed line is obtained by least square adjustment, with slope 1.46 .

have built the INPOP05 ephemeris. Few elements differ between INPOP05 and DE405.

\subsubsection{Sun and Solar System barycenter}

In JPL planetary ephemerides, the Sun is not integrated in the same way as the planets. Its position and velocity are determined from those of the planets and asteroids, assuming that the Solar System barycenter (SSB) remains at the origin of the inertial reference frame. If $\boldsymbol{r}_{i}$ is the barycentric position, $\boldsymbol{v}_{i}$ the barycentric velocity vector, and $m_{i}$ the mass of body $i$, it is assumed that (see Le Poncin-Lafitte et al. 2006)

$\sum_{i} \mu_{i}^{*} \boldsymbol{r}_{i}=\mathbf{0}$

with $\mu_{i}=G m_{i}$ and up to order $1 / c^{2}$,

$\mu_{i}^{*}=\mu_{i}\left(1+\frac{v_{i}^{2}}{2 c^{2}}-\frac{1}{2 c^{2}} \sum_{j \neq i} \frac{\mu_{j}}{r_{i j}}\right)$.

By derivation of (1) we have

$\sum_{i} \mu_{i}^{*} \dot{\boldsymbol{r}}_{i}+\dot{\mu}_{i}^{*} \boldsymbol{r}_{i}=\mathbf{0}$

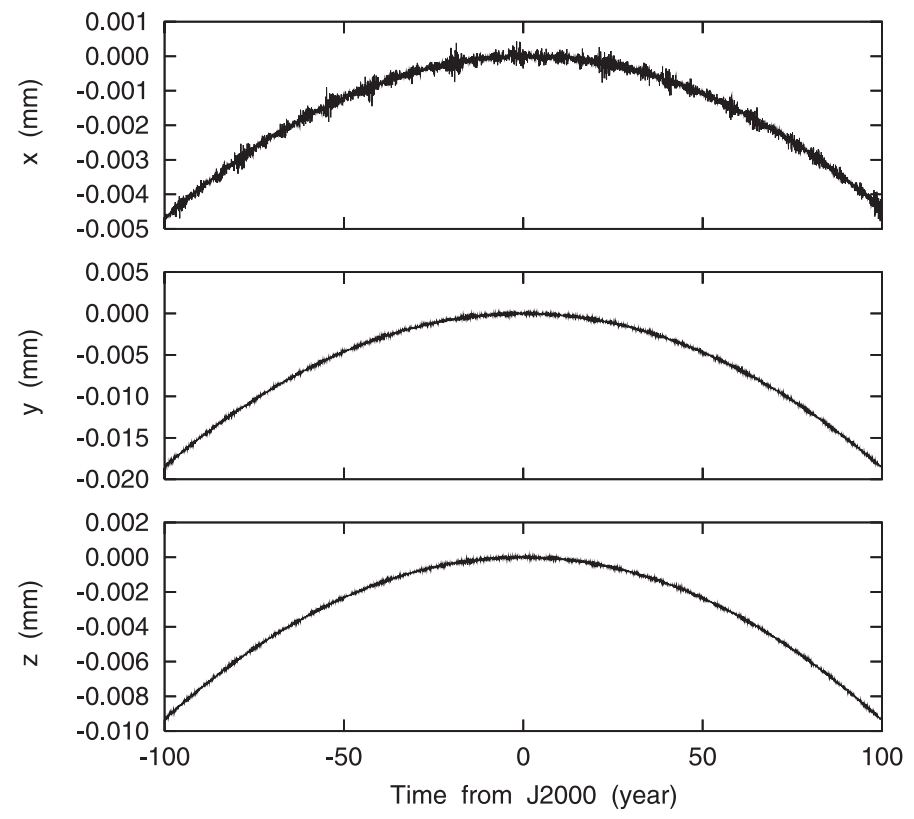

Fig. 3. These curves show the drift of SSB in INPOP. We consider here a simplified model, composed of the Sun, the planets from Mercury to Pluto and the Moon (no asteroids), all considered as point-mass bodies. At the time origin of integration (J2000), the origin $O$ of the reference frame is at the barycenter $G$ of the system (Eqs. $(2,4,5))$. The curves show the drift of the barycenter $G$ in the reference frame, that is $\boldsymbol{O} \boldsymbol{G}=\left(\sum \mu_{i}^{*}\right)^{-1} \sum_{i} \mu_{i}^{*} \boldsymbol{r}_{i}$. Time interval is in years from $\mathbf{J} 2000$ and the coordinates of $G$ are in mm.

with, by derivation of (2) and up to order $1 / c^{2}$,

$\dot{\mu}_{i}^{*}=\frac{\mu_{i}}{2 c^{2}}\left(\sum_{j \neq i} \mu_{j} \frac{\left(\boldsymbol{r}_{j}-\boldsymbol{r}_{i}\right) \cdot\left(\dot{\boldsymbol{r}}_{j}+\dot{\boldsymbol{r}}_{i}\right)}{r_{i j}^{3}}\right)$.

In INPOP, we have taken the term $\dot{\mu}_{i}^{*} \boldsymbol{r}_{i}$ into account. Moreover, the Sun is treated as the other planets without assuming a fixed SSB. Indeed, because of the approximations that are still made in the computation of $\mu_{i}^{*}$ and $\mu_{i}^{*}$, there remains a small drift of the SSB in the fixed reference frame centered on the SSB at J2000. The determination of the SSB at the origin of time (J2000) is obtained by solving the equations

$$
\left\{\begin{array}{l}
\sum_{i} \mu_{i}^{*} \boldsymbol{r}_{i}=\mathbf{0} \\
\sum_{i} \mu_{i}^{*} \dot{\boldsymbol{r}}_{i}+\dot{\mu}_{i}^{*} \boldsymbol{r}_{i}=\mathbf{0}
\end{array}\right.
$$

where $\mu_{i}^{*}$ and $\mu_{i}^{*}$ are given by Eqs. (2) and (4). Because $\mu_{i}^{*}$ and $\dot{\mu}_{i}^{*}$ depend on the planet velocities, an iterative process is needed. Contrarily to the JPL method, these equations are solved only at the initial step of the planetary integration, at J2000. Once the frame is centered on the SSB defined by Eqs. (5) at J2000, the equations of motion of planets and Sun are integrated in this fixed reference frame. Because of the approximations in $1 / c^{2}$, the positions and velocities of the SSB deduced at $t$ still has a very small displacement that can be neglected (Fig. 3).

If, as in the JPL model (Standish 2004), the $\mu_{i}^{*}$ term is neglected in the second equation of (5), a more important drift appears in the SSB motion (Fig. 4). Although this does not have any impact on the precision of the solutions, we have preferred to keep the $\mu_{i}^{*}$ term in INPOP for better consistency. 

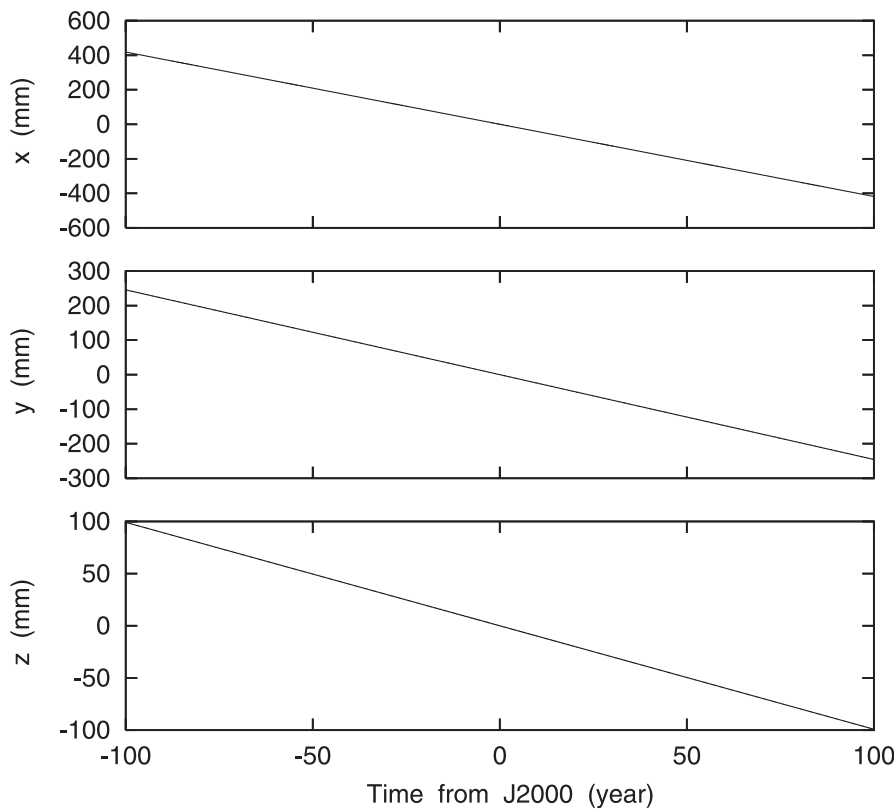

Fig. 4. Same kind of simulation as for Fig. 3, but in this case, $\mu_{i}^{*}$ is neglected in (5) when computing the SSB at the time origin of integration.

\subsubsection{Asteroids}

INPOP05 uses the same asteroid set as DE405 does. In INPOP05, all asteroid orbits are numerically integrated, taking into account the planetary perturbations on asteroids.

Once the asteroid positions and velocities are obtained, and in order to remain very close to the DE405 model, the INPOP05 computation of perturbations on planets does not differ from DE405: only asteroid perturbations upon Mars, the Earth and the Moon are taken into account for 297 of them (see Sect. 2.4.1 for more details).

\subsubsection{Earth tides}

In DE102 (Newhall et al. 1983) and DE200, only tides raised by the Moon on the Earth are considered (Newhall et al. 1983). In INPOP, as DE403 and followers, Sun tides are added and the Earth shape varies with time: Earth coefficients of the potential are variable parameters. Expressions of their variations are given in Lambeck (1988).

$$
\left\{\begin{array}{l}
\Delta C_{20}=\frac{m_{p}}{M}\left(\frac{R}{r_{p}^{*}}\right)^{3} \frac{k_{20}}{2} \frac{2 r_{z}^{* 2}-r_{x}^{* 2}-r_{y}^{* 2}}{r_{p}^{* 2}} \\
\Delta C_{21}=\frac{m_{p}}{M}\left(\frac{R}{r_{p}^{*}}\right)^{3} k_{21} \frac{r_{x}^{*} r_{z}^{*}}{r_{p}^{* 2}} \\
\Delta C_{22}=\frac{m_{p}}{M}\left(\frac{R}{r_{p}^{*}}\right)^{3} \frac{k_{22}}{4} \frac{r_{x}^{* 2}-r_{y}^{* 2}}{r_{p}^{* 2}} \\
\Delta S_{21}=\frac{m_{p}}{M}\left(\frac{R}{r_{p}^{*}}\right)^{3} k_{21} \frac{r_{y}^{*} r_{z}^{*}}{r_{p}^{* 2}} \\
\Delta S_{22}=\frac{m_{p}}{M}\left(\frac{R}{r_{p}^{*}}\right)^{3} \frac{k_{22}}{2} \frac{r_{x}^{*} r_{y}^{*}}{r_{p}^{* 2}} .
\end{array}\right.
$$

In these equations, $m_{p}$ is the mass of the tide rising body (Sun or Moon), $M$ and $R$ are respectively the mass and the mean equatorial radius of the Earth, $k_{2 m}$ is the Love number associated to the harmonic $2 m$. The vector $\boldsymbol{r}_{p}^{*}$ of the Earth-tide rising body is estimated with a time delay $\tau_{2 m}$, depending on the order of the harmonic $2 m: \boldsymbol{r}_{p}^{*}=\boldsymbol{r}_{p}\left(t-\tau_{2 m}\right) . \boldsymbol{r}_{p}^{*}$ is given in the Earth's frame.

\subsubsection{Earth orientation}

When interactions with the figure of the Earth are computed, the knowledge of the Earth orientation is needed. This is the case for the computation of interactions between the non-spherical Earth and point-mass bodies, the computation of the deformation of the Earth and also for the computation of the torque exerted on the Moon due to the interaction between the non-spherical Earth and the non-spherical Moon. As in DE405, in INPOP05 the orientation of the Earth's axis is obtained with the precession model of Williams (1994) and the nutation expression of Wahr (1981), limited to the main $18.6 \mathrm{yr}$ term.

\subsubsection{Moon deformations}

As for the Earth, in INPOP, the Moon is assumed to be a deformable body, shaped by its own rotation and the tides raised by the Earth. The Moon potential coefficients are time varying and the same coefficient developments as in Sect. 2.3.3 are used. However, Love numbers and time delay are assumed to be independent of the order of the harmonic. Furthermore, as in DE405, variations of the Moon inertia tensor due to the Moon rotation is estimated following Newhall et al. (1997). Additional variations of the coefficients of potential are then

$$
\left\{\begin{array}{l}
\Delta C_{20}=\frac{k R^{3}}{3 G M} \frac{1}{2}\left(\omega^{* 2}+2 n^{2}-3 \omega_{z}^{* 2}\right) \\
\Delta C_{21}=-\frac{k R^{3}}{3 G M} \omega_{x}^{*} \omega_{z}^{*} \\
\Delta S_{21}=-\frac{k R^{3}}{3 G M} \omega_{y}^{*} \omega_{z}^{*} \\
\Delta C_{22}=\frac{k R^{3}}{3 G M}\left(\omega_{y}^{* 2}-\omega_{x}^{* 2}\right) \\
\Delta S_{22}=-\frac{k R^{3}}{3 G M} \frac{1}{2} \omega_{x}^{*} \omega_{y}^{*} .
\end{array}\right.
$$

In these expressions, $k$ is the Love number (the same for all harmonics), $R$ is the mean equatorial radius, $G$ is the gravitational constant, $M$ is the mass of the Moon, $\omega^{*}$ is the instantanous vector of rotation (estimated with a time delay $\tau$, that is $\left.\omega^{*}(t)=\omega(t-\tau)\right)$ and $n$ is the mean motion of the Moon around the Earth.

\subsubsection{Comparison to DE405}

In order to analyze the differences between DE405 and the INPOP05 model, we have first integrated INPOP05 with the same initial conditions and parameters as for DE405. Using the initial conditions and constant values given in the DE405 header, we integrate INPOP05 over the whole time interval of DE405 ( $-400 \mathrm{yr}$ to $200 \mathrm{yr}$ with origin at J2000). The maximum differences obtained between DE405 and INPOP05 are shown in Table 2. The agreement between the two ephemerides is very good, especially for the estimation of the lunar geocentric positions. The differences, except for Mars, are in general much smaller than the residual of the comparisons with the observations (see Sect. 4.2). For Mars, the differences are significantly larger than for the other planets. This may be due to the asteroid orbits which are computed in a slightly different way in DE405 and INPOP05 or INPOP06. In Table 2, the maximum differences 
Table 2. Maximum difference between DE405 and INPOP05 (with the same initial conditions) in range $(r)$, latitude $(\phi)$ and longitude ( $\lambda$. If $(x, y, z)$ are the heliocentric (geocentric for the Moon) coordinates of the planet in the equatorial reference frame of the ICRF, then $r=\sqrt{\left(x^{2}+y^{2}+z^{2}\right)}, \phi=\arcsin (z / r)$ and $\lambda=\arctan (y / x)$. EMB is the Earth-Moon Barycenter. For the librations of the Moon, $\phi, \theta$ and $\psi$ are the usual Euler's angles (see Newhall et al. 1983, Sect. II.D). Comparisons are made over the whole time interval of DE405 ( $-400 \mathrm{yr}$ to $200 \mathrm{yr}$ with origin at J2000); Col. 1: from $-30 \mathrm{yr}$ to $+30 \mathrm{yr}$; Col. 2 : from $-100 \mathrm{yr}$ to $+100 \mathrm{yr}$; Col. 3 : from $-400 \mathrm{yr}$ to $200 \mathrm{yr}$.

\begin{tabular}{|c|c|c|c|}
\hline \multicolumn{4}{|c|}{ Heliocentric range $r(\mathrm{~m})$} \\
\hline Mercury & 6.2 & 26 & 228 \\
\hline Venus & 0.5 & 2.1 & 18 \\
\hline EMB & 1.6 & 7.7 & 60 \\
\hline Mars & 58 & 517 & 3365 \\
\hline Jupiter & 31 & 108 & 467 \\
\hline Saturn & 20 & 36 & 121 \\
\hline Uranus & 38 & 45 & 69 \\
\hline Neptune & 35 & 77 & 80 \\
\hline Pluto & 31 & 119 & 283 \\
\hline Moon (geocentric) & 0.009 & 0.034 & 0.540 \\
\hline \multicolumn{4}{|c|}{ Longitude $\lambda$ ( $\mu$ as) } \\
\hline Mercury & 174 & 749 & 6637 \\
\hline Venus & 78 & 534 & 6270 \\
\hline EMB & 32 & 286 & 2494 \\
\hline Mars & 354 & 6600 & 42070 \\
\hline Jupiter & 57 & 276 & 1012 \\
\hline Saturn & 16 & 51 & 201 \\
\hline Uranus & 4 & 14 & 41 \\
\hline Neptune & 0.7 & 14 & 39 \\
\hline Pluto & 0.9 & 7 & 44 \\
\hline Moon (geocentric) & 60 & 170 & 6173 \\
\hline \multicolumn{4}{|c|}{ Latitude $\phi$ ( $\mu$ as) } \\
\hline Mercury & 65 & 268 & 2373 \\
\hline Venus & 30 & 207 & 2262 \\
\hline EMB & 13 & 113 & 979 \\
\hline Mars & 152 & 2602 & 16970 \\
\hline Jupiter & 23 & 102 & 383 \\
\hline Saturn & 5 & 17 & 67 \\
\hline Uranus & 0.6 & 5 & 15 \\
\hline Neptune & 0.2 & 5 & 14 \\
\hline Pluto & 0.4 & 3 & 19 \\
\hline Moon (geocentric) & 25 & 73 & 2478 \\
\hline \multicolumn{4}{|c|}{ Lunar librations ( $\mu$ as) } \\
\hline$\phi$ & 900 & 900 & 1000 \\
\hline$\theta$ & 400 & 400 & 400 \\
\hline$\psi$ & 800 & 800 & 5800 \\
\hline
\end{tabular}

between DE405 and INPOP05 for the three libration angles can be found for several intervals of time. In this case, again, the agreement between the two ephemerides is very satisfactory.

\subsection{A new dynamical model: INPOP06}

Once we have verified, with INPOP05, that we are able to match very closely the JPL DE405 ephemerides, we construct the new model INPOP06 that will differ more significantly from the DE405 model. We searched for a dynamical model that follows our best understanding of the planetary and rotational dynamics of the Solar System, with the aim to reach the accuracy of the observations that will be available with the next space missions (Venus Express, GAIA, Bepi Colombo...). We have also searched for an ephemeris that is as self consistent as possible, avoiding input of factors that are computed in a separate manner (the precession of the Earth, for example). We thus have different options for the Earth orientation and deformation, and for asteroid perturbations.

\subsubsection{Asteroids}

As INPOP05, INPOP06 sees asteroids as planet-like. Their orbits are numerically integrated with the planets.

The same 300 asteroids are used in INPOP06 and INPOP05, however the computation of their perturbations on planets differs. In INPOP05 and DE405, only the perturbations induced by Ceres, Pallas and Vesta are taken into account for all the planets. The other 297 perturbations are summed and used only for the Earth, the Moon and Mars orbit computations.

In INPOP06, the 300 asteroids are divided into 2 groups. In the first one, 5 asteroids (Ceres, Pallas, Vesta, Iris and Bamberga) are considered exactly like the planets by taking into account mutual perturbations, perturbations of the planets and of the 295 other asteroids, and solar oblateness in the PPN formulism (Moyer 1971). The second group contains the remaining 295 asteroids. For them, only Newtonian perturbations of the planets and of the 5 asteroids for the first group are considered. The main difference to DE405 is that with INPOP06, the perturbations of all the 300 asteroids upon all the planets are taken into account $^{1}$. As Fienga \& Simon (2005) have shown, this induces significant drifts in the inner planet orbits. Fit to observations of the 5 largest asteroid masses (Ceres, Pallas, Vesta, Iris and Bamberga) and of the 3 taxonomic densities (C, S, and $\mathrm{M}$ ) are made in INPOP06.

The Krasinsky et al. (2002) proposition of adding an asteroid ring potential in the inner planet orbit computations is also extended to the outer planets.

The asteroid ring is assumed to be cicular, with radius $r_{0}$ and mass $M$, and centered on the Solar System barycenter. The perturbed body with barycentric position vector $\boldsymbol{r}$ is supposed to be in the ring plane (the mutual inclination of the planets is neglected). Let $(\boldsymbol{u}, \boldsymbol{v})$ be a direct orthogonal basis in the ring plane, with $\boldsymbol{u}=\boldsymbol{r} / r$, and let $\boldsymbol{r}^{\prime}(\theta)=r_{0}(\cos \theta \boldsymbol{u}+\sin \theta \boldsymbol{v})$ be the position vector of a point of the ring. The acceleration of the body due to the ring is:

$\ddot{\boldsymbol{r}}=\frac{G M}{2 \pi} \int_{0}^{2 \pi} \frac{\boldsymbol{r}^{\prime}(\boldsymbol{\theta})-\boldsymbol{r}}{\left\|\boldsymbol{r}^{\prime}(\boldsymbol{\theta})-\boldsymbol{r}\right\|^{3}} \mathrm{~d} \theta$.

Expressed in $(\boldsymbol{u}, \boldsymbol{v})$, one obtains:

$\ddot{\boldsymbol{r}}=\frac{G M}{2 \pi} \int_{0}^{2 \pi} \frac{\left(r_{0} \cos \theta-r\right) \boldsymbol{u}+r_{0} \sin \theta \boldsymbol{v}}{\left(r^{2}+r_{0}^{2}-2 r r_{0} \cos \theta\right)^{3 / 2}} \mathrm{~d} \theta$.

For an inner body $\left(r<r_{0}\right)$, (9) can be expanded in Fourier series using Laplace coefficients ${ }^{2}$ and after averaging over $\theta$, one finaly obtains:

$\ddot{\boldsymbol{r}}=\frac{G M}{2 r r_{0}^{2}}\left(b_{3 / 2}^{(1)}\left(r / r_{0}\right)-\frac{r}{r_{0}} b_{3 / 2}^{(0)}\left(r / r_{0}\right)\right) \boldsymbol{r}$.

1 The perturbations of the asteroids on all the main planets have also been taken into account in DE414 (Konopliv et al. 2006) and beginning with EPM2000 (Pitjeva 2001).

2 The Laplace coefficients $b_{s}^{k}(\alpha)$ are defined as the coefficients of the Laurent series (see Laskar 2005)

$(1-\alpha z)^{-s}\left(1-\alpha z^{-1}\right)^{-s}=\frac{1}{2} \sum_{k=-\infty}^{+\infty} b_{s}^{(k)}(\alpha) z^{k}$. 

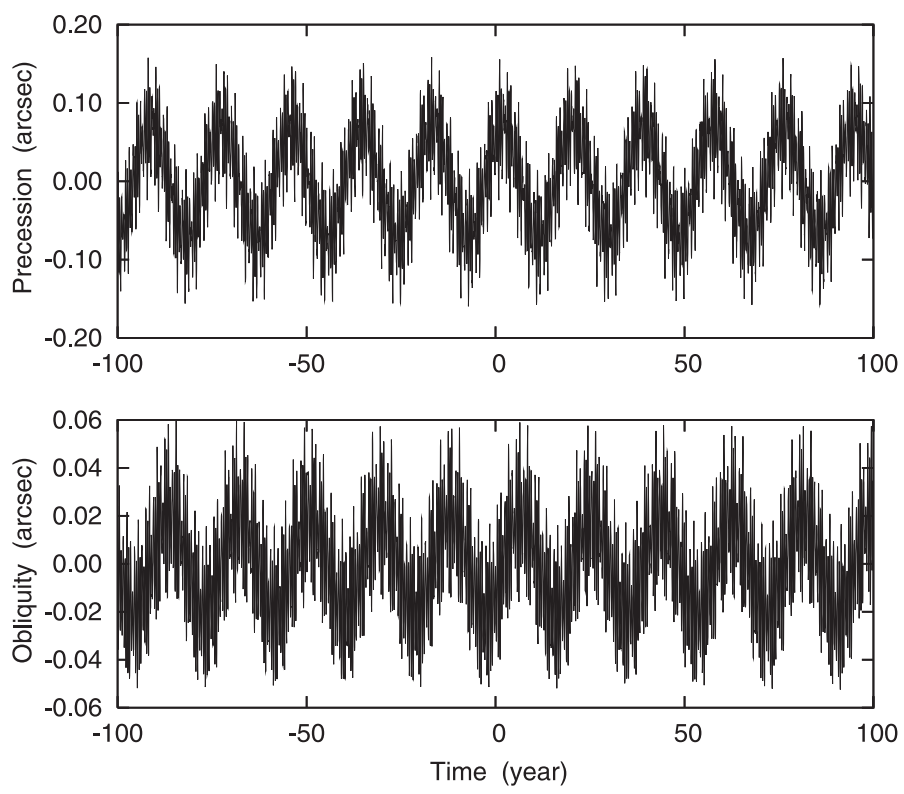

Fig. 5. Discrepancies in the Earth's orientation between INPOP06 and the CIP-P03 in precession and obliquity (units are in arcsec). Comparisons are made over the interval $-100 \mathrm{yr}$ to $+100 \mathrm{yr}$ with the origin at $\mathbf{J} 2000$.

This expression is equivalent to the one given in Krasinsky et al. (2002) in term of hypergeometric functions. For an outer body $\left(r>r_{0}\right)$, the same developpements lead to the expression of the acceleration of an outer body due to the asteroid ring:

$\ddot{\boldsymbol{r}}=\frac{G M}{2 r^{3}}\left(\frac{r_{0}}{r} b_{3 / 2}^{(1)}\left(r_{0} / r\right)-b_{3 / 2}^{(0)}\left(r_{0} / r\right)\right) \boldsymbol{r}$.

In INPOP06, the mass of a circular asteroid ring with $2.8 \mathrm{AU}$ radius centered at the Solar System barycenter is fitted to observations.

\subsubsection{Earth deformations}

The Earth deformations are modeled as described in Sect. 2.3.3. Secular variation of the Earth $J_{2}$, due to the postglacial rebound of the mantle, is also taken into account in the computation of the acceleration and torques applied on the Earth with constant rate $\left(\dot{J}_{2}=-3 \times 10^{-9} / c y\right)$. This value was determined by satellite laser ranging (Yoder et al. 1983) and is used by Williams (1994) and by Capitaine et al. (2005) in the precession model P03 that has been recently adopted by the IAU (IAU 2006 resolution 1$)^{3}$.

\subsubsection{Earth orientation}

In INPOP06, the Earth orientation is integrated at the same time as all other bodies and Moon libration. With INPOP05 and DE405, the Earth orientation was estimated with Williams (1994) and Wahr (1981) precession and nutation models. These two models are based on some planetary ephemerides, and are expressed in term of periodic and polynomial terms that are valid only over a few thousand years. Then, inconsistencies between

\footnotetext{
${ }^{3}$ In the P03 model, $\dot{J}_{2}=-3.001 \times 10^{-9} / c y$, but the $0.001 \times 10^{-9} / c y$ difference is meaningless because of the very coarse relative precision of the determination of this value. Indeed, a significant change in $\dot{J}_{2}$ has been recently observed (Cox et al. 2002).
}

the Earth orbital and rotational motion can appear. In order to allow longer time integrations, and ensure the self-consistency of the solutions, we have chosen to integrate the rotation of the Earth together with its orbital motion.

The numerical integration of the full rotational motion of the Earth would require a very small step size, based on the rotational frequency of the Earth $(\omega)$. Following Boué \& Laskar (2006), we have chosen here to average over the rotational motion of the Earth. Indeed, if $A, B, C$ are the principal momenta of inertia of the Earth, $\boldsymbol{K}$ is the unit vector in the direction of the largest momentum of inertia $C$, and $\boldsymbol{w}=\boldsymbol{G} /\|\boldsymbol{G}\|$ the unit vector in the direction of the rotational angular momentum of the Earth, the averaged value $\langle\boldsymbol{K}>$ of $\boldsymbol{K}$ is extremely close to $\boldsymbol{w}$. From Boué \& Laskar (2006),

$<\boldsymbol{K}>=\boldsymbol{w}+O\left(J^{2}\right)$,

where $J$ is the angle between $\boldsymbol{K}$ and $\boldsymbol{w}$. From the solution for a rigid Earth SMART97 (Bretagnon et al. 1998), it can be deduced that for the Earth, $|J|<1.22 \times 10^{-7} \mathrm{rad}$ and $J^{2}=O\left(10^{-14}\right)$. The orientation vector $\boldsymbol{K}$ can thus be replaced by the $\boldsymbol{w}=\boldsymbol{G} /\|\boldsymbol{G}\|$ angular momentum vector. The evolution of the angular momentum, up to term in $O\left(J^{2}\right)$ is given by Boué \& Laskar (2006)

$\dot{\boldsymbol{G}}=3 \frac{2 C-(A+B)}{2\|\boldsymbol{G}\|^{2}} \sum_{i} \frac{\mu_{i}}{r_{i}^{5}}\left(\boldsymbol{r}_{i} \cdot \boldsymbol{G}\right) \boldsymbol{r}_{i} \wedge \boldsymbol{G}$,

where $\boldsymbol{r}_{i}$ are the position vector of the perturbing bodies $(i=$ $1, \ldots 9$ is the index for the Moon, Sun and all planets except the Earth from Mercury to Neptune). All quantities are expressed in the fixed frame of integration. The initial conditions for the unit vector $\boldsymbol{w}=\boldsymbol{G} /\|\boldsymbol{G}\|$ and form parameter $C / M R^{2}$ (where $C$ is the largest moment of inertia, $M$ the mass, and $R$ the mean equatorial radius of the Earth) are fitted on the CIP-P03 pole in the ICRF reference frame (Capitaine et al. 2005) over 200 years around J2000. The fitted value for $C / M R^{2}$ can be found in Table 5 .

Figure 5 shows the differences between the integration of the Earth's orientation with INPOP06 and the CIP-P03. The differences, smaller than 0.2 arcsec in the angle of precession and 0.07 arcsec in obliquity, are due to the "free core nutations", not taken into account in our integration. We compare here the mean angular momentum vector $\boldsymbol{w}$ integrated in INPOP06 with the CIP-03 that is an approximation of the attitude vector $\boldsymbol{K}$. The differences of $\boldsymbol{w}$ with the true angular momentum of the Earth would be much smaller as the angular momentum is not affected (at first order) by the liquid core contribution.

Over time intervals longer than 1000 years, the differences between the computed Earth orientation in INPOP06 and the CIP-P03 start to diverge significantly (Fig. 7). This results from the presence of polynomial approximations in the precession angles of the CIP-P03. Beyond about 2000 years, the errors in the secular terms of the CIP-P03 precession formulas will exceed the short period discrepancies between INPOP06 and CIP-P03 resulting from the consideration of the liquid core in CIP-P03 (Fig. 5).

\subsubsection{Remarks}

Our main reason for integrating the spin axis of the Earth is to obtain a solution that is not limited in time, and can thus be extended to several thousands and even millions of years. Indeed, as seen in Fig. 7, beyond about 2000 years, our solution of the Earth axis becomes more precise than the CIP-03 precession and obliquity solution. In particular, the INPOP06 solution for the 

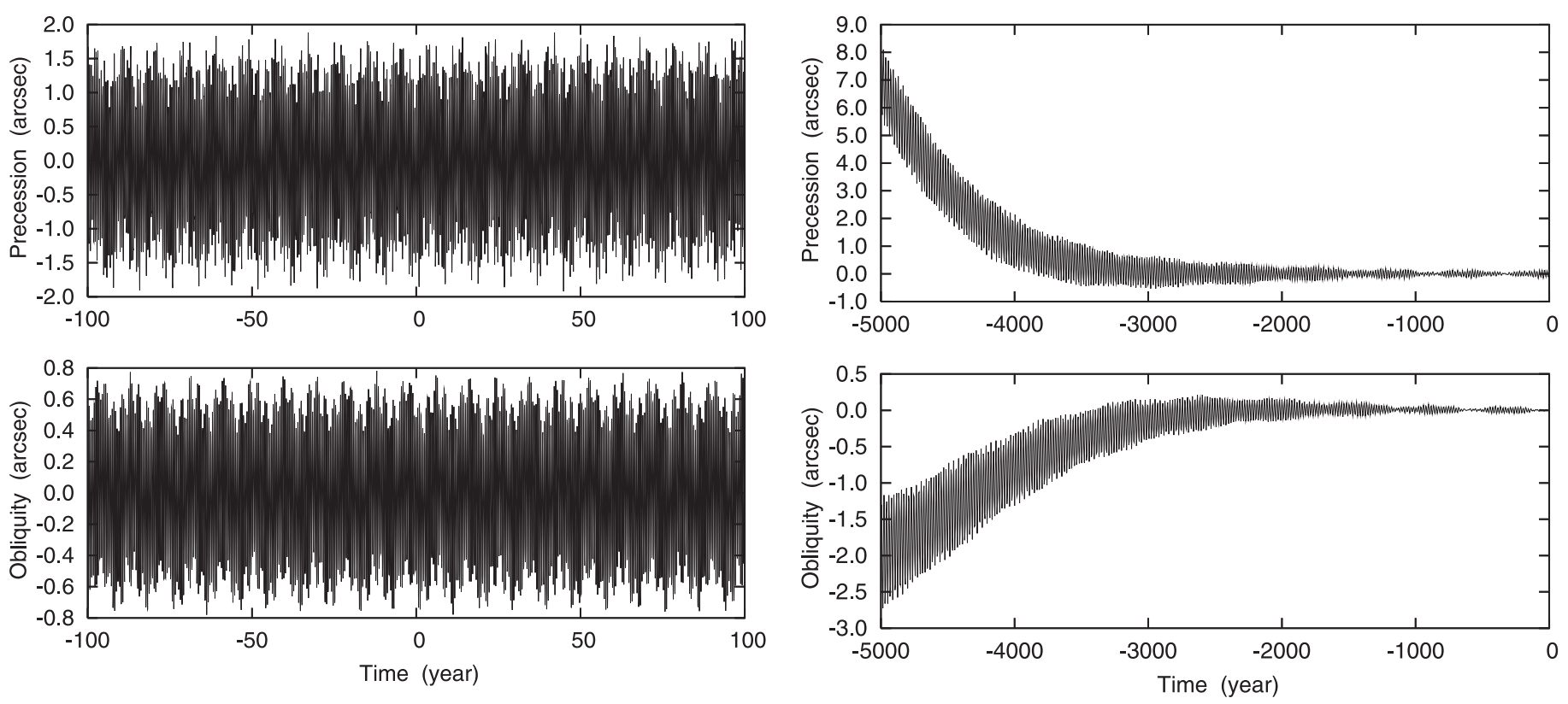

Fig. 6. Discrepancies in Earth's orientation between the Earth's pole used to compute DE405 (precession of Williams 1994 and the single $18.6 \mathrm{yr}$ nutation term from Wahr 1981) and the CIP-P03 in precession and obliquity (units are in arcsec). Comparisons are made over the interval $-100 \mathrm{yr}$ to $+100 \mathrm{yr}$ with origin at $\mathrm{J} 2000$.

Earth axis is an independent way to verify the CIP-03 precession.

The approximation of the attitude vector of the Earth $\boldsymbol{K}$ that we obtain by computing $\boldsymbol{w}$ is more accurate than the approximation of $\boldsymbol{K}$ that is presently used in the JPL ephemerides models (Fig. 6), although both models are sufficiently precise for the computation of orbital ephemerides.

In the reduction process of the observations, that is over time span limited to a few tens of years, solutions and parameters of the Earth rotation consistent with the ICRF definition are used: precession-nutation of the IAU2000 (Mathews et al. 2002; Chapront et al. 2002), and Earth orientation parameters provided by the IERS. The algorithm is based on the SOFA routines (Wallace 2004).

\section{Planetary ephemerides overview}

Since 1998, the JPL DE405 ephemerides has been the worldwide reference for planetary ephemerides. It is used at JPL and ESA to prepare space missions. As demonstrated by Standish \& Fienga (2002), the uncertainty on the main belt asteroid masses induces inaccuracies on the Mars heliocentric and geocentric orbits. However several Mars missions took place since 1998 (Pathfinder, Mars Global Surveyor, Mars Odyssey) providing new information that can be used to better constrain asteroid perturbations. Several new ephemerides including these data were then built by JPL (DE410, DE414), by IAA RAS (EPM2000, EPM2004), and by IMCCE (INPOP06). The main differences between these ephemerides are the asteroid perturbation modeling and the Mars observations used in the fit. In DE410, perturbations induced by a ring were applied on Mars and Earth orbits. The observational interval for DE410 fit is from 1899 to January 2004, including MGS data. In DE414, in addition to ring perturbations, more than 64 asteroid masses were estimated with the DE414 fit including MGS and Mars Odyssey data until

Fig. 7. Discrepancies in the Earth's orientation between INPOP06 and the CIP-P03 in precession and obliquity (units are in arcsec). Comparisons are made over $-5000 \mathrm{yr}$ with the origin at J2000.

April 2005. As far as we know, DE414 is the most advanced JPL ephemeris for Mars.

\section{INPOP observational processing and adjustment}

The complete data processing dedicated to planetary ephemerides fitting was presented in Standish (1990). However, we discuss here some differences in the INPOP processing of data.

Three main types of observations are used for planetary ephemerides fits: direct radar observations of the planet surface (Venus, Mercury and Mars), spacecraft tracking data (radar ranging, Doppler and VLBI), and optical observations (transit, photographic plates and CCD observations for outer planets).

\subsection{Observational processing}

\subsubsection{Mercury and Venus}

For Mercury and Venus, most of the observations are direct radar observations. The signal is emitted by an antenna and reflected directly by the planet surface. This kind of observation is not very accurate because it is strongly correlated with our knowledge of the planet topography. Usually, the uncertainties of these observations are a few kilometers.

To correct the data for surface topography, we use for Venus the Rapaport \& Plaut (1994) model based on the Magellan observations, and for Mercury, the Anderson et al. (1996) model based on radar observations of the Mercury surface done from 1967 to 1990 . The INPOP fit is based on JPL observations carried out from 1971 to 1997 for Mercury and from 1964 to 1990 for Venus.

For direct radar observations and for ranging data from spacecraft (MGS, Odyssey), solar plasma corrections are needed. Here, one can fit correction coefficients during the ephemerides adjustement (Pitjeva 2001, 2005). 
For INPOP, the Anderson et al. (2005) solar corona modeling is applied. This model includes the latest estimation of solar electronic density from the Cassini mission. The relativistic correction is given by Moyer (1971). When a tropospheric correction is needed, we applied the simplified model given by Standish (1990).

VLBI observations of the Venus orbiter, named Magellan, were obtained in the early nineties (Folkner 1992, 1993, 1994a,b). They are VLBI measurements of the spacecraft with respect to background sources from a radio source catalogue. Thanks to these observations, it is possible to tie the inner planet system to the ICRF radio frame.

\subsubsection{Mars}

For Mars, different types of observations are involved. In the INPOP fit, we choose to take into account only the space tracking data arguing that the accuracy from a few kilometers (for older ones) to $100 \mathrm{~m}$ for the direct radar observations of the Mars surface can be ignored compared to the few meters accuracy of the modern space observations.

Viking, Pathfinder, MGS and Mars Odyssey observations were used in the INPOP06 fit. Only Viking data were used for the INPOP05 fit. These missions have furnished orbiter ranging, lander ranging (Viking and Pathfinder missions) and differenced range, and orbiter VLBI observations (MGS mission).

For direct radar observations and spacecraft ranging, the procedure is very similar. In principle, relativistic, tropospheric and plasma (solar corona) corrections are applied in the same manner. However, for spacecraft tracking data, some corrections (usually the tropospheric correction, and sometimes the plasma correction) were applied by the navigation teams.

The orbiter ranging data are distances furnished by the JPL navigation teams, which means that they are free from spacecraft orbits. They are distances from the Mars center of mass to the antenna at the Earth surface. The reduction to the geocenter is done in the IERS frame, using IAU 2000 recommendations for precession, nutation and Earth orientation parameters (see Sect. 2.4.4).

The processing of the Viking and Pathfinder lander ranging data are more complex because the data are distances from the Mars surface where the lander is situated to the Earth surface where the antenna is located. Mars rotation models must then be introduced. Some publications such as EPM (Pitjeva 2005) give fitted rotation model parameters and lander coordinates obtained during the planetary ephemerides fit. For INPOP, we use the values of the Mars rotation parameters and Mars nutation model given by Standish (2001). The lander coordinates are the one computed by JPL (Standish 2001).

The processing of the lander Doppler observations corresponds to differenced radar range observations. Differences are computed between two round-trip times observed at $60 \mathrm{~s}$ time interval. Estimations of variations of the Mars lander to Earth antenna distances are then obtained. Because such observations could be seen as radial velocity estimations, they are usually called Doppler observations. The same models as the one described previously for Mercury, Venus and Mars ranging are used, including the Mars rotation correction induced by the lander location. The description of the Viking and Pathfinder differenced range is given in Folkner et al. (1997).

MGS and Mars Odyssey VLBI data were obtained during the orbit of the spacecraft to Mars. Here again, these data are differenced VLBI data. Spacecraft angular positions versus the observed radio sources are estimated. Since the spacecraft orbit is well known, the navigation teams deduced angular positions of planets versus the reference radio sources. These data, with the Venus Magellan and the Jupiter Galileo observations, establish the relationships between the dynamical frame based on INPOP planetary ephemerides and the ICRF.

\subsubsection{Outer planets}

To link the INPOP Jupiter plane to the same ICRF reference plane as the inner planets, 44 VLBI differenced observations obtained during the Galileo missions are used in the Jupiter orbit fit. These data were also provided by JPL navigation teams. A complete description can be found in Folkner (1998).

For Jupiter and Saturn, besides previous direct optical observations of the planets, observations of satellites are taken into account in the fit. A major source of systematic errors in the outer planet astrometry is the phase effect. Due to the lightening gradient and the scattering law, the determination of the center of mass relative to the photocenter is very difficult. Several methods were tested to correct for these effects (Fienga \& Delouis 2001; Fienga 1999) but better accuracies are obtained from satellite observations (Fienga 1998). As their surfaces are telluric and their apparent diameters are much smaller, models of the phase effect are easier and most of the time the satellite phase defect is neglected. The dynamical theories of satellites used in INPOP are those published by Vienne \& Duriez (1995) and Arlot (1982). The combination of accurate relative positions of satellites and observed right ascension and declination of good quality allows us to obtain accurate equatorial coordinates of the planets. The problem of the phase effect of the planets is then removed.

For Uranus, Neptune and Pluto, we used direct observations of the planets. Considering the accuracy reached by the transit, photographic and CCD observations, phase effects can be neglected. For Pluto VLT observations, this assumption is not true and phase effect correction must be applied.

Common treatments related to reference frames are applied to all optical observations. As the INPOP plane of reference must be linked with ICRF, each outer planet optical observations are expressed in the ICRF following IAU 2000 recommendations. Depending on the publication frames of the data, different algorithms are applied. For old observations, corrections from FK3 to FK4 and FK4 to FK5 frames are applied (Yallop 1989; Frike 1971). FK5 zonal corrections are also taken into account (Schwan 1988) and rotations from the FK5 to the ICRF (Mignard \& Froeschlé 1998) are applied. Such transformations guarantee, at the level of accuracy of the optical data (about 100 mas), the link between the INPOP outer planet frame and ICRF. Galileo VLBI observations orbiting Jupiter enforce this link at the VLBI accuracy which means a factor of 100 improvement compared to the optical observations tie.

\subsection{INPOP05 fit to observations}

In order to validate our fitting process, we first fitted INPOP05 with the same samples of observations as those used to obtain DE405. Postfit residuals are shown in Table 3.

For Mercury and Venus, no topography model is fitted but the model described in Sect. 4.1.1 is used. With Mars, no fit of the Viking lander coordinates is done and no direct radar observations of Mars are included in the fit. For the outer planets, the fit includes direct observations of the planet photocenter and positions deduced from satellite observations. As in DE405, 
Table 3. INPOP05 (Col. 5) residuals for each type of observations. Column 3 gives the time interval of observations and Col. 4 the number $N$ of observations used in the fit and in the residual computations. For optical observations, the residuals are given respectively in right ascension and declination $(\alpha ; \delta)$. The given uncertainties are at 1-sigma.

\begin{tabular}{lllccc}
\hline \hline Planet & Type of data & Time interval & $N$ & $\begin{array}{c}\text { INPOP05 } \\
\langle(o-c)\rangle\end{array}$ & \multicolumn{1}{c}{$\sigma$} \\
& & & & -520.0 & 951 \\
Mercury & Radar [m] & $1971-1998$ & 415 & -1717.0 & 4058 \\
Venus & Radar [m] & $1964-1990$ & 737 & -175 & 1375 \\
& & after 1965 & 488 & 590 & \\
Spacecraft VLBI [mas] & $1990-1994$ & 18 & 1.6 & 3 & 12.5 \\
Mars & Vkg lander radar [m] & $1976-1983$ & 1253 & 0.37 & $(480 ; 472)$ \\
Jupiter & optical $(\alpha, \delta)[\mathrm{mas}]$ & $1914-1994$ & 2872 & $(-32 ; 472)$ & $(-504 ; 454)$ \\
Saturn & optical $(\alpha, \delta)[\mathrm{mas}]$ & $1914-1994$ & 2339 & $(-52 ;-19)$ & $(504)$ \\
Uranus & optical $(\alpha, \delta)[\mathrm{mas}]$ & $1914-1994$ & 2909 & $(50 ; 9)$ & $(400 ; 401)$ \\
Neptune & optical $(\alpha, \delta)[\mathrm{mas}]$ & $1914-1994$ & 2830 & $(78 ;-52)$ & $(436 ; 396)$ \\
Pluto & optical $(\alpha, \delta)[\mathrm{mas}]$ & $1989-1993$ & 208 & $(-62 ;-18)$ & $(270 ; 255)$ \\
\hline
\end{tabular}
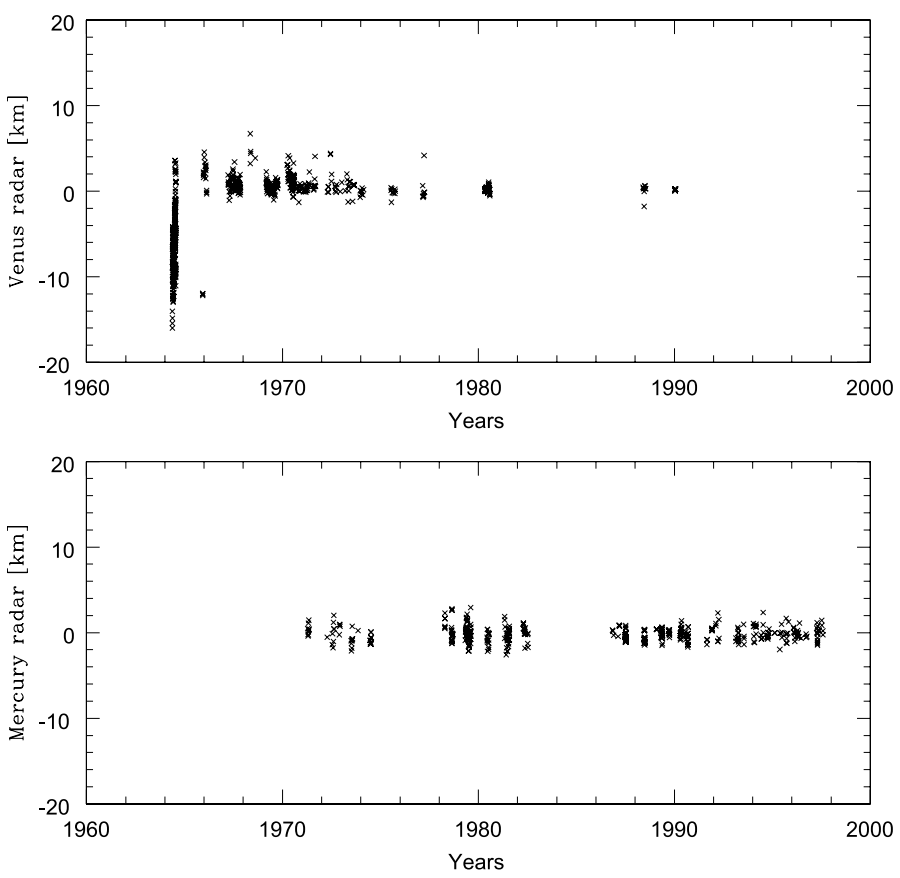

Fig. 8. Mercury and Venus direct radar observation comparison to INPOP06. $y$-axis is given in kilometers and $x$-axis is dates.

besides the planet initial conditions, 3 asteroid masses and 3 asteroid taxonomic classes are fitted to observations.

\section{INPOP06}

After building a twin ephemeris of DE405 (see Sect. 2.3), dynamical modeling improvements are carried and a fit was possible with all available observations. A new independent planetary ephemeris, called INPOP06, was then built. We describe in the following sections the fit to the observations.

\subsection{New features in observational fit}

The fit of INPOP06 was performed using observations until June 2005.

In addition to the planet initial conditions, we also fit 5 asteroid masses, 3 taxonomic classes, the ring mass (its distance to the Sun is fixed to $2.8 \mathrm{UA}$ ) and the Sun oblateness (J2). Fitted physical parameters are presented in Table 7. Different values extracted from other planetary ephemerides are also presented in this table. One can find the values obtained with DE414, DE405 and EPM2004.

\subsection{INPOP06 after fit}

The maximum differences between INPOP06 fitted to observations and DE405 are presented in Table 4 over several intervals of time.

For the planets, the differences are important due to the changes in the dynamical model (asteroid perturbations over all the planets, use of an asteroid ring) and to the fit made with new observations. Especially for Mars, the differences in heliocentric longitudes induce a difference of about $200 \mathrm{~m}$ in geocentric distances. Such an effect is mainly due to the more complex modeling of the asteroid perturbations and to the observational fit carried out on MGS/Odyssey data. These data are five time more accurate than the Viking observations, which were the most accurate spacecraft data used in the Mars DE405 fitting and thanks to the MGS/Odyssey sample, the Mars space missions observational time interval is now extended to over 30 years.

In INPOP06, the Moon orbit and rotation modelings are the same as in INPOP05. However, as modifications were done in the INPOP06 planet dynamical model and a new observational fit was made, the Moon ephemerides changed. To stay close to the DE/LE405 ephemerides of lunar motion (which is fitted on LLR observations), a fit of the Moon geocentric initial conditions and time delays ( $\tau_{21}$ and $\tau_{22}$ for the Earth, $\tau$ for the Moon) was done on the DE/LE405 Earth-Moon distance. In Table 4, one may see that the differences between DE/LE405 and INPOP06 Moon ephemerides stay close in geocentric longitude, latitude and distance as well as in libration angles. The values of the time delays deduced from the fit of the INPOP06 Moon and the ones used for DE/LE405 can be found in Table 5.

In Table 6, we give the INPOP06 postfit residuals. Figures 810 show the residuals obtained with INPOP06 after the fit. The values in Table 6 give an estimation of the accuracy reached by INPOP06 after it has been fitted to observations.

\subsection{Fit of physical parameters}

In Table 7, the fitted values of physical parameters computed by INPOP06 are gathered. Values extracted from other planetary ephemerides are also shown. One can see the high consistency of the values between different ephemerides. The helioseismic 
Table 4. Maximum difference between DE405 and INPOP06 in range $(r)$, latitude $(\phi)$ and longitude $(\lambda)$. $(x, y, z)$ are the heliocentric (geocentric for the Moon) coordinates of the planet in the equatorial reference frame of the ICRF, and $r=\sqrt{\left(x 2+y^{2}+z^{2}\right)}, \phi=\arcsin (z / r)$ and $\lambda=\arctan (y / x)$. EMB is the Earth-Moon Barycenter. For the librations of the Moon, $\phi, \theta$ and $\psi$ are the usual Euler's angles (see Newhall et al., section II.D). Comparisons are made over the whole time interval of DE405 ( $-400 \mathrm{yr}$ to $200 \mathrm{yr}$ with origin at J2000); Col. 1: from -30 yr to $+30 \mathrm{yr}$; Col. 2 : from $-100 \mathrm{yr}$ to $+100 \mathrm{yr}$; Col. 3 : from $-400 \mathrm{yr}$ to $200 \mathrm{yr}$.

\begin{tabular}{|c|c|c|c|}
\hline \multicolumn{4}{|c|}{ Heliocentric range $r(\mathrm{~m})$} \\
\hline Mercury & 500 & 1000 & 2800 \\
\hline Venus & 51 & 63 & 84 \\
\hline EMB & 19 & 31 & 67 \\
\hline Mars & 102 & 360 & 5000 \\
\hline Jupiter & 19000 & 31000 & 94000 \\
\hline Saturn & 290000 & 290000 & 350000 \\
\hline Uranus & 890000 & 1000000 & 1500000 \\
\hline Neptune & 2600000 & 3200000 & 3300000 \\
\hline Pluto & 9100000 & 35000000 & 68000000 \\
\hline Moon (geocentric) & 0.007 & 0.08 & 1.8 \\
\hline \multicolumn{4}{|c|}{$\bar{L}$ Longitude $\lambda(\mu$ as) } \\
\hline Mercury & 11000 & 21000 & 53000 \\
\hline Venus & 1600 & 2200 & 2200 \\
\hline EMB & 1600 & 2100 & 3200 \\
\hline Mars & 1500 & 5400 & 63000 \\
\hline Jupiter & 46000 & 140000 & 530000 \\
\hline Saturn & 130000 & 180000 & 440000 \\
\hline Uranus & 240000 & 350000 & 1200000 \\
\hline Neptune & 160000 & 650000 & 1200000 \\
\hline Pluto & 240000 & 1500000 & 12000000 \\
\hline Moon (geocentric) & 1700 & 2700 & 28000 \\
\hline \multicolumn{4}{|c|}{ Latitude $\phi$ ( $\mu$ as) } \\
\hline Mercury & 4200 & 6700 & 22000 \\
\hline Venus & 690 & 890 & 900 \\
\hline EMB & 1800 & 1800 & 1800 \\
\hline Mars & 1700 & 3200 & 25000 \\
\hline Jupiter & 21000 & 55000 & 210000 \\
\hline Saturn & 73000 & 86000 & 170000 \\
\hline Uranus & 150000 & 240000 & 400000 \\
\hline Neptune & 84000 & 160000 & 470000 \\
\hline Pluto & 110000 & 670000 & 4900000 \\
\hline Moon (geocentric) & 1800 & 1900 & 12000 \\
\hline \multicolumn{4}{|c|}{ Lunar librations ( $\mu$ as) } \\
\hline $\bar{\phi}$ & 7700 & 7900 & 7900 \\
\hline$\theta$ & 3900 & 3900 & 3900 \\
\hline$\psi$ & 6800 & 6800 & 26000 \\
\hline
\end{tabular}

determination of Pijpers (1998), $J_{2}=2.18 \pm 0.06 \times 10^{-7}$, is inside the errorbars of our fitted value of $J_{2}\left(2.46 \pm 0.40 \times 10^{-7}\right)$. The INPOP determination of the Sun oblateness $J_{2}$ was estimated with the fixed standard values for the PPN parameters ( $\beta=\gamma=1$ ), but we have also performed some tests on the $\beta$ determinations during the INPOP06 fit to observations. With the fixed value of the Sun oblateness $J_{2}=2.46 \times 10^{-7}$, the best residuals are obtained with $|\beta-1|<10^{-5}$. On the other hand, if $J_{2}$ and $\beta$ are considered as free parameters, then the best combination for residual minimization is $J_{2}=2.97 \times 10^{-7} \pm 0.40$ and $|\beta-1|<$ $10^{-4}$. As one can see in Table 8 , the results are consistent with the values obtained with the planetary ephemerides EPM2004 and EPM2006 (Pitjeva 2005, 2006). Values for EPM2004 are also the upper bounds with free solution parameters $J_{2}, \beta$ and $\gamma$. In Table 8, the given upper bounds determined from INPOP06, EPM2004 and EPM2006 for $J_{2}$ and $|\beta-1|$ are maximum values
Table 5. Parameters used in DE405 (and INPOP05) (Col. 2), and in INPOP06 (Col. 3), to compute the tidal effects (see Sects. 2.3.3 and 2.3.5) and the Earth orientation. $\tau_{E 21}$ and $\tau_{E 22}$ are respectively the Earth's time delays for harmonics $(2,1)$ and $(2,2)$, and $\tau_{\mathrm{M}}$ is the time delays for the Moon. They are expressed in days and rounded to $10^{-10}$. $\mathrm{C}, \mathrm{M}$ and $\mathrm{R}$ are respectively the maximum moment of inertia, the mass and the mean equatorial radius of the Earth.

\begin{tabular}{ccc}
\hline \hline Constants & DE405 & INPOP06 \\
\hline$\tau_{E 21}$ & 0.0129089594 & 0.0129385159 \\
$\tau_{E 22}$ & 0.0069417856 & 0.0069390352 \\
$\tau_{\mathrm{M}}$ & 0.1667165558 & 0.1668420986 \\
$C / M R 2$ & & 0.330822078 \\
\hline
\end{tabular}
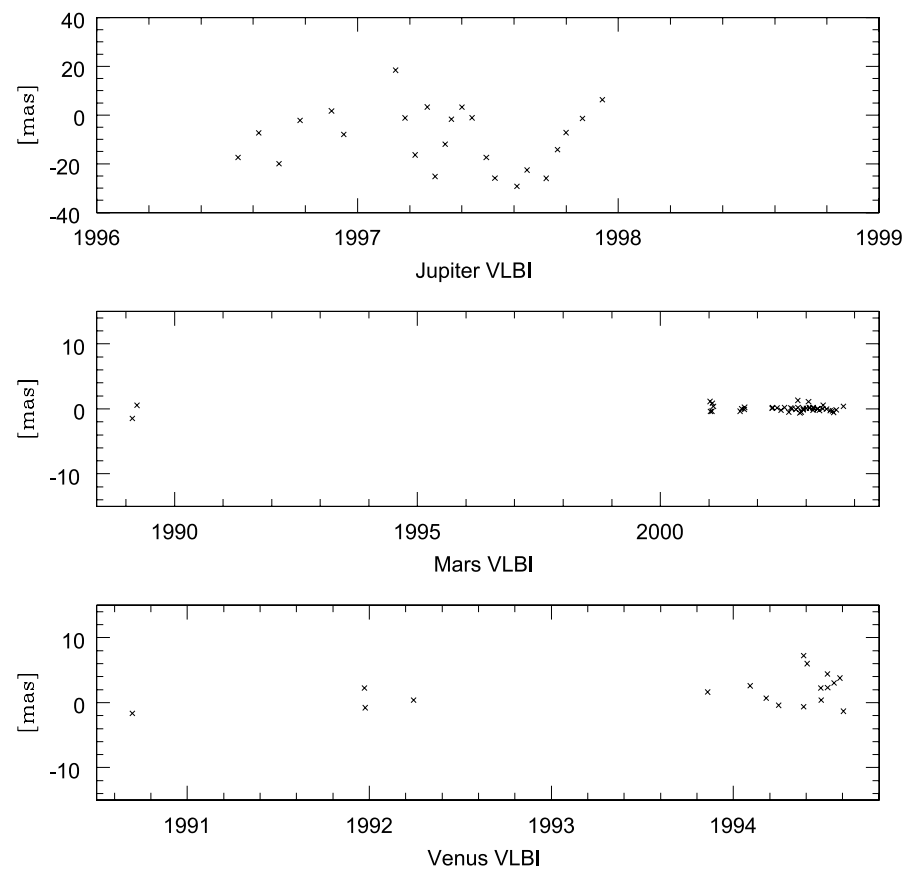

Fig. 9. Venus, Mars and Jupiter VLBI observation comparison to INPOP06. $y$-axis is given in mas and $x$-axis is dates.

of stability deduced from several parameter estimations minimizing the planetary observations residuals. These values give thus the upper limit of the stability zone of the determination. The determinations of $\beta$ with INPOP06 are significantly more precise than the values published by Will (2006), obtained during the reduction process of other kinds of data (spacecraft time delays and VLBI observations).

\section{Future developments and conclusions}

We have presented our work on the construction of a new independent numerical ephemeris for the planet and Sun motions as well as Earth and Moon rotations. We have introduced the dynamical models used for the description of planetary motion and rotations. We have shown how we analyzed planetary observations and how we fit INPOP ephemerides to observations. Prefit and postfit comparisons between the numerically integrated orbits and the observed positions are presented. The results were obtained by minimizing the number of fitted parameters. Only values of direct physical parameters not related to observational methods are determined from the INPOP06 

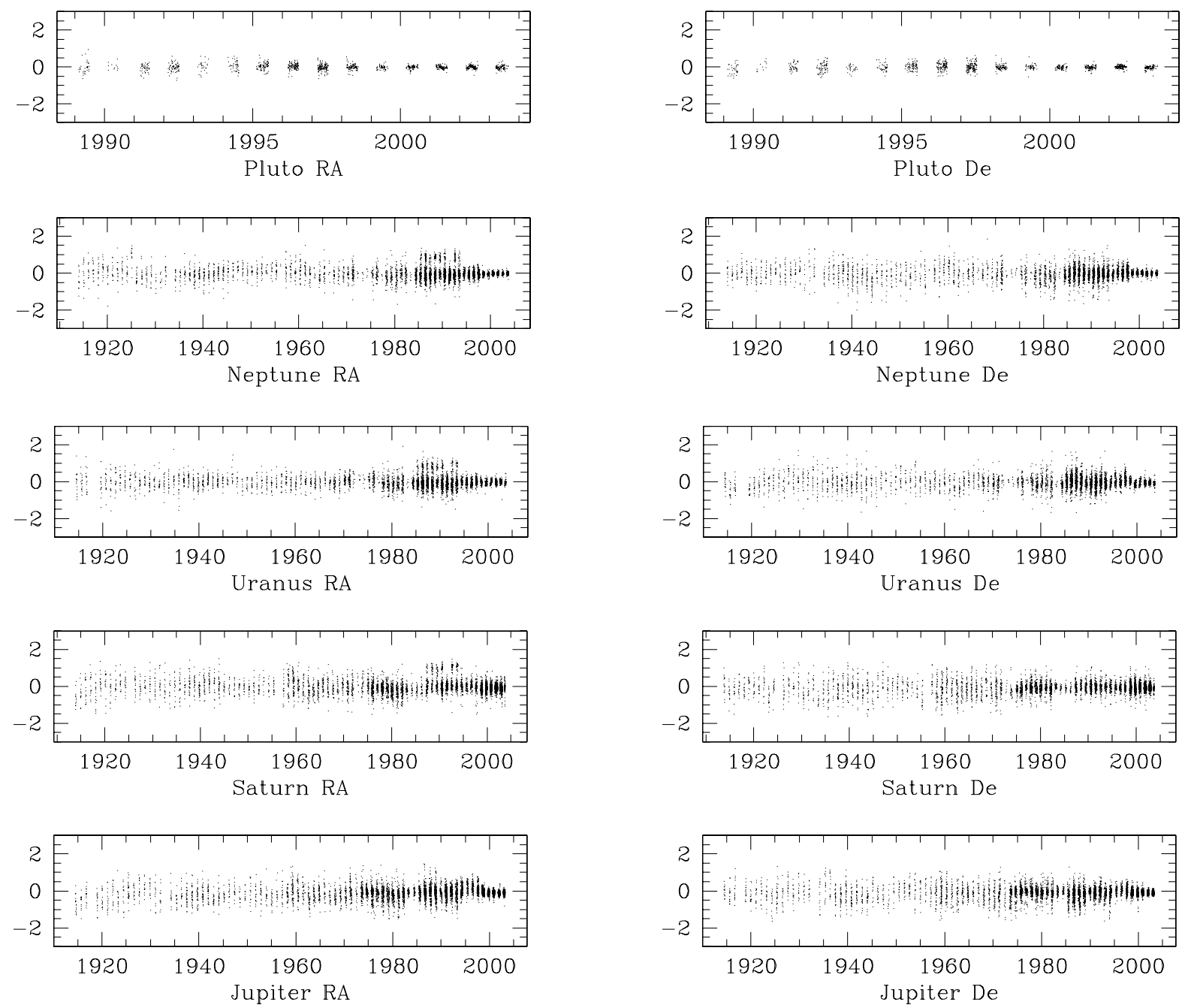

Fig. 10. Outer planet optical observations to INPOP06. $y$-axis is given in arcseconds and $x$-axis is date.

fit. A public release of INPOP06 is available on the website http: //www . imcce. fr/inpop with a Tchebychev polynomial representation compatible with the JPL programs using JPL tchebychev files. An INPOP06 realization of the TCB time scale will be published soon.

Several new aspects and improvements will be investigated for the next INPOP version.

The Moon orbital ephemerides and libration should be fitted directly to Lunar Laser Ranging (LLR) observations. LLR data will be used to estimate new initial conditions and libration parameters as well as tests for new inner Moon models. Furthermore, a new LLR station based on adaptative optics (APOLLO) has begun to obtain very accurate observations (Murphy et al. 2002). Such new observations are very promising for a better understanding of the lunar dynamics and inner physics. In the same way as an inner Moon model has to be introduced, the INPOP Earth orientation could be improved by the introduction of a liquid core. With this addition, an improvement of about a factor ten could be expected in the comparisons between INPOP Earth orientation and P03.

Analysis of new observations based on the European tracking of the MEX and Venus Express (VEX) missions will bring new information related to the very accurate modeling of Mars and Venus orbits. New tests on the asteroid selection of perturbing objects are also to be done. The classification of the asteroids in three taxonomic classes can be improved. A new organization of asteroid families according to their dynamical properties has to be tested. The VEX observations will be very important because since the Magellan mission in the nineties, no accurate data have been obtained for the Venus orbit.

In order to densify the sets of data used for the reference frame tie, a new link to the ICRF can be attempted using millisecond pulsar timings. Progress in pulsar timing observations and reduction procedures (Hobbs et al. 2006) will make thus these data interesting in the Earth orbit fit. The construction of a pulsar catalogue linked to ICRF by pulsar VLBI observations can indirectly improve the orientation of the INPOP planetary ephemerides with respect to the ICRF.

Acknowledgements. We thank M. Standish for multiple dicussions during this work. N. Capitaine and all the participants of the working group on planetary ephemerides (GTEP) held at Paris Observatory are thanked for their input. The authors especially thank the ESA/ESOC navigation team of the interplanetary missions MEX and VEX for their help and explanations. This work was supported by CNES under contract 05/CNES//00-DCT 094, by the CS of Paris Observatory, and by PNP-CNRS. 
Table 6. INPOP06 (Col. 5) residuals for each type of observation. Column 3 gives the observational time interval and Col. 4 the number of observations $N$ used in the fit and in the residual computations. For optical observations, the residuals are given respectively in right ascension and declination $(\alpha ; \delta)$. The uncertainties are at 1-sigma.

\begin{tabular}{lllccc}
\hline \hline Planet & Type of data & Time interval & $N$ & $\begin{array}{c}\text { INPOP06 } \\
\langle(o-c)\rangle\end{array}$ & \multicolumn{1}{c}{$\sigma$} \\
\hline Mercury & Radar [m] & $1971-1998$ & 444 & -239 & 855 \\
Venus & Radar [m] & $1964-1990$ & 737 & -1727 & 4051 \\
& & after 1965 & 488 & 583 & 1385 \\
\multirow{4}{*}{ Mars } & Spacecraft VLBI [mas] & $1990-1994$ & 18 & 1.7 & 2 \\
& Vkg lander radar [m] & $1976-1983$ & 1256 & -23 & 18 \\
& MGS/Odyssey radar [m] & $1999-2005.45$ & 10474 & 4.0 & 5.5 \\
& Vkg Doppler [mm/s] & $1976-1979$ & 1501 & -0.26 & 4.4 \\
& Pathfinder Doppler [mm/s] & 1997 & 1519 & -0.34 & 0.97 \\
Jupiter & Spacecraft VLBI [mas] & $1989-2003$ & 44 & 0.4 & 0.5 \\
& Spacecraft VLBI [mas] & $1996-1998$ & 24 & -9 & 12 \\
Saturn & optical $(\alpha, \delta)$ [mas] & $1914-2004$ & 5536 & $(-17 ;-24)$ & $(341 ; 331)$ \\
Uranus & optical $(\alpha, \delta)$ [mas] & $1914-2004$ & 5573 & $(-6 ; 13)$ & $(347 ; 311)$ \\
Neptune & optical $(\alpha, \delta)$ [mas] & $1914-2004$ & 3848 & $(12 ; 10)$ & $(357 ; 366)$ \\
Pluto & optical $(\alpha, \delta)$ [mas] & $1914-2004$ & 3898 & $(11 ; 12)$ & $(368 ; 356)$ \\
\hline
\end{tabular}

Table 7. Physical parameters fitted in INPOP06. Other values deduced from planetary ephemerides are presented for comparisons.The given uncertainties are given at 1-sigma.

\begin{tabular}{llrrrr}
\hline \hline & Unit & DE405 & EPM2004 & DE414 & INPOP06 \\
\hline & & & & & \\
Mass of Ceres & $10^{-10} M_{\odot}$ & 4.64 & $4.753 \pm 0.007$ & $4.699 \pm 0.006$ & $4.756 \pm 0.004$ \\
Mass of Vesta & $10^{-10} M_{\odot}$ & 1.34 & $1.344 \pm 0.001$ & $1.358 \pm 0.004$ & $1.348 \pm 0.003$ \\
Mass of Pallas & $10^{-10} M_{\odot}$ & 1.05 & $1.027 \pm 0.003$ & $1.026 \pm 0.006$ & $1.025 \pm 0.001$ \\
Mass of Iris & $10^{-10} M_{\odot}$ & & $0.063 \pm 0.001$ & $0.060 \pm 0.002$ & $0.058 \pm 0.001$ \\
Mass of Bamberga & $10^{-10} M_{\odot}$ & & $0.055 \pm 0.001$ & $0.047 \pm 0.002$ & $0.046 \pm 0.003$ \\
Mass of Ring & $10^{-10} M_{\odot}$ & & $3.35 \pm 0.35$ & $0.31 \pm 0.27$ & $0.34 \pm 0.15$ \\
Distance of Ring & $\mathrm{UA}$ & & $3.13 \pm 0.05$ & 2.8 & 2.8 \\
Density of the C class & & 1.8 & $1.5 \pm 0.03$ & $1.62 \pm 0.07$ & $1.56 \pm 0.02$ \\
Density of the S class & & 2.4 & $2.2 \pm 0.04$ & $2.08 \pm 0.19$ & $2.18 \pm 0.04$ \\
Density of the M class & & 5.0 & $3.84 \pm 0.12$ & $4.32 \pm 0.37$ & $4.26 \pm 0.12$ \\
Sun J2 & $10^{-7}$ & 2 & $1.9 \pm 0.3$ & $2.34 \pm 0.49$ & $2.46 \pm 0.40$ \\
\hline
\end{tabular}

Table 8. Solar oblateness and the PPN parameter $\beta$ determinations based on INPOP06 fit to observations. In the first column, the publications from where the values are extracted and the methods used to estimate the Sun J2 and the PPN parameters $\beta$ are given. In Cols. 2, 3 and 4 , computed values of these parameters can be found.

\begin{tabular}{lll}
\hline \hline & $\begin{array}{l}\text { Sun J2 } \\
10^{-7}\end{array}$ & $\begin{array}{l}|\beta-1| \\
10^{-5}\end{array}$ \\
\hline $\begin{array}{l}\text { This paper } \\
\text { INPOP06 }\end{array}$ & $\begin{array}{l}2.46 \\
2.97 \pm 0.40\end{array}$ & $<1$ \\
INPOP06 & $<10$ \\
(Pitjeva 2005) & & \\
$\begin{array}{l}\text { EMP2004 } \\
\text { (Pitjeva 2006) }\end{array}$ & $1.9 \pm 0.3$ & $(0 \pm 10)$ \\
EMP2006 & $2 \pm 0.5$ & $<20$ \\
$\begin{array}{l}\text { (Will 2006) } \\
\text { Heliosismology }\end{array}$ & $2.2 \pm 0.1$ & $(0 \pm 300)$ \\
\hline
\end{tabular}

\section{References}

Anderson, J. D., Jurgens, R. F., Lau, E. L., Slade, M. A., III, \& Schubert, G. 1996, Icarus, 124, 690

Anderson, J. D., Laing, P. A., Lau, E. L., et al. 2005,

[arXiv:astroph/0104064]

Arlot, J.-E. 1982, A\&A, 107, 305
Ash, M. E., Shapiro, I. I., \& Smith, W. B. 1971, Science, 174, 551

Boué, G., \& Laskar, J. 2006, Icarus, 185, 2

Bretagnon, P., Francou, G., Rocher, P., \& Simon, J.-L. 1998, A\&A, 329, 329

Brouwer, D. 1937, AJ, 46, 149

Capitaine, N., Wallace, P. T., \& Chapront, J. 2005, A\&A, 432, 355

Chapront, J., Chapront-Touzé, M., \& Francou, G. 2002, A\&A, 387, 700

Cox, C. M., \& Chao, B. 2002, Science, 297, 831

Devine, C. J., \& Dunham, D. W. 1966, 33, 232

Fienga, A. 1998, A\&A, 335, 111

Fienga, A. 1999, BAAS, 31, 1589

Fienga, A., \& Delouis, J. M. 2001, A\&A, 368, 1115

Fienga, A., \& Simon, J.-L. 2005, A\&A, 429, 361

Folkner, W. M. 1992, JPL IOM, 335.1, 92

Folkner, W. M. 1993, 335.1, 93

Folkner, W. M. 1994a, JPL IOM, 335.1, 94

Folkner, W. M. 1994b, 335.1, 94

Folkner, W. M., Kahn, R. D., Preston, R. A., et al. 1997, J. Geophys. Res., 102, 4057

Fricke, W. 1971, A\&A, 13, 298

Hairer, E., Nordsett, S., \& Wanner, G. 1993, Solving Ordinary Differential Equations, Vols. I, Second Ed. (Springer-Verlag)

Hobbs, G. B., Edwards, R. T., \& Manchester, R. N. 2006, MNRAS, 369, 655

Konopliv, A. S., Yoder, C. F., Standish, E. M., Yuan, D.-N., \& Sjogren, W. L. 2006, Icarus, 182, 23

Krasinsky G. A., Pitjeva E. V., Sveshnikov M. L., \& Sveshnikova E. S. 1982, Bul. Inst. Theoret. Astron. 15, N3

Krasinsky, G. A., Aleshkina, E. Yu., Pitjeva, E. V., \& Sveshnikov, M. L. 1986, IAU Symp., 114, ed. J. Kovalevsky, V. A. Brumberg, D. Reidel (Dordrecht: Publ. Com.), 315 
Krasinsky, G. A., Pitjeva, E. V., Sveshnikov, M. L., \& Chunajeva, L. I. 1993, Celest. Mech., 55, 1

Krasinsky, G. A., Pitjeva, E. V., Vasilyev, M. V., \& Yagudina, E. I. 2002, Icarus, 158,98

Lambeck, K. 1988, Geophysical geodesy (Clarendon Press)

Laskar, J. 1989, Nature, 338, 237

Laskar, J. 1990, Icarus, 88, 266

Laskar, J. 2005, Celest. Mech., 91, 351

Laskar, J., Correia, A. C. M., Gastineau, M., et al. 2004a, Icarus, 170, 343

Laskar, J., Robutel, P., Joutel, F., et al. 2004b, A\&A, 428, 261

Le Poncin-Lafitte, C., Manche, H., Fienga, A., \& Laskar, J. 2006, private communication

Markstein, P. 2000, IA-64 and Elementary Functions (New Jersey: Prentice Hall)

Mathews, P. M., Herring, T. A., \& Buffet, B. A. 2002, Modeling of nutation and precession: New nutation series for nonrigid Earth and insights into the Earth's interior, J. Geophys. Res., 107, B4

Mignard, F., \& Froeschlé, M. 1998, Highlights Astron., 11, 313

Moyer, T. 1971, JPL Tech. Report, 32, 157

Murphy, T. W., Aldelberg, E. G., Strasburg, J. D., \& Stubbs, C. W. 2002, in Thirteenth International Workshop on Laser Ranging, Washington D. C., on October 07 through 11, 2002

Newhall, X. X., \& Williams, J. G. 1997, Celest. Mech. Dyn. Astron., 66, 21

Newhall, X. X., Standish, E. M., \& Williams, J. G. 1983, A\&A, 125, 150

Pijpers, F. P. 1998, MNRAS, 297, L76
Pitjeva, E. V. 2001, Celest. Mech. Dyn. Astron., 80, 249

Pitjeva, E. V. 2005, Sol. Syst. Res., 39, 176

Pitjeva, E. V. 2006, Nomenclature, Precession and New Models in Fundamental Astronomy 26th meeting of the IAU, Joint Discussion 16

Quinn, T., \& Tremaine, S. 1990, AJ, 99, 1016

Rappaport, N., \& Plaut, J. J. 1994, Icarus, 112, 27

Schwan, H. 1988, A\&A, 198, 363

Standish, E. M. 1990, A\&A, 233, 272

Standish, E. M. 1995, JPL IOM, 314.10, 127

Standish, E. M. 1998, JPL IOM, 312.F, 98

Standish, E. M. 2001, private communication

Standish, E. M. 2003, JPL IOM, 312.N, 03

Standish, E. M. 2004, A\&A, 41, 1165

Standish, E. M. 2005, private communication

Standish, E. M., \& Fienga, A. 2002, A\&A, 384, 322

Standish E. M. Jr., Keesey M. S., \& Newhall XX 1976, JPL Technical 32, 1603

Vienne, A., \& Duriez, L. 1995, A\&A, 297, 588

Wahr, J. M. 1981, Geophys. J. Roy. Astron. Soc. 64, 705

Wallace, P. T. 2004, Am. Astron. Soc. Meet., 20428.02

Will, C. M. 2006, Living Rev. Relativity, 9, 3

Williams, J. G. 1994, AJ, 108, 711

Yallop, B. D., Hohenkerk, C. Y., Smith, C. A., et al. 1989, AJ, 97, 274

Yoder, C. F., Dickey, J. O., Schultz, B. E., Eanes, R. J., \& Tapley, B. D. 1983, Nature, 303, 391 